\title{
Accumulation of Focal Adhesion Protein Hic-5 in the Nucleus by Hydrogen Peroxide
}

\author{
Motoko Shibanuma $^{1}$, Keiko Ishino ${ }^{1}$, Nobuko Sakamoto ${ }^{1}$ and Kiyoshi Nose ${ }^{1}$ \\ ${ }^{1}$ Department of Microbiology, Showa University School of Pharmaceutical Sciences, Hatanodai 1-5-8, \\ Shinagawa-ku, Tokyo 142-8555
}

Received April 3, 2001; accepted May 21, 2001

\begin{abstract}
Reactive oxygen species (ROS) are produced in cultured cells upon stimulation with cytokines and tumor promoters, and participate in signal transduction pathways leading to gene expression. The hic-5 gene was isolated as a hydrogen peroxide-inducible clone by differential screening, and was shown to encode a paxillin-related protein localized in the focal adhesion plaques. In mouse osteoblastic and fibroblastic cells treated with low concentrations of hydrogen perox-

ide, Hic-5 protein was found to be reversibly localized to the nucleus within $30 \mathrm{~min}$. Hic-5 was also translocated to the nucleus in cells treated with leptomycin B, an inhibitor of nuclear export of proteins. Hic-5 protein with mutations in the nuclear export signal and the LIM-3 domain accumulated in the nucleus. These results indicated that Hic-5 shuttles between the cytoplasm and the nucleus, and may participate both in surface signals and in gene expression.
\end{abstract}

Key words: Hic-5, Nuclear translocation, NES, Hydrogen peroxide

\section{Introduction}

Reactive oxygen species (ROS) including superoxide anion, hydroxy radicals, hydrogen peroxide and lipid peroxides are produced in various types of cells in response to various signals. Harmful ROS appear to be trapped mostly by intracellular or extracellular scavenging substances, but there is accumulating evidence of some important functions of ROS in signaling pathways and the regulation of cellular phenotypes. The signals participate in normal physiological responses as well as in aging or pathological disorders.

The essential roles of ROS and derived species in signal transduction were reviewed by Lander [9] and Finkel [3]. Extracellular ligands such as hormones and cytokines trigger activation of cytoplasmic signals that lead to nuclear events through a cascade of protein-protein interactions. Recent studies indicate that many extracellular ligands generate and/or require ROS or derived species to transmit their signals to the nucleus $[11,16,18]$. TGF $\beta 1$ treatment activates production of hydrogen peroxide from osteoblastic cells, and hydrogen peroxide participates in the induction of egr-1 gene expression [16]. The CArG box present upstream of the egr-1 gene was essential for induction. Platelet-derived

Correspondence to: Kiyoshi Nose, Department of Microbiology, Showa University School of Pharmaceutical Sciences, Hatanodai 1-58, Shinagawa-ku, Tokyo 142-8555, Japan. growth factor also stimulated smooth muscle cells to produce hydrogen peroxide, which is involved in MAP kinase activation [18]. Phorbol 12-myristate 13-acetate induced MCP-1/JE gene transcription that was dependent on active oxygen species, and $\mathrm{NFKB}$ was required for the induction [11].

During the course of our studies to identify signaling molecules that participate in the ROS cascade, we isolated a novel gene, hic-5, by differential screening of a cDNA library from mouse osteoblastic cells [17]. The hic-5 gene encodes a polypeptide of about $50 \mathrm{kDa}$ with a marked similarity to paxillin, a well known component of the focal adhesion complex. Hic-5 protein is co-localized in the focal adhesion complex with vinculin and paxillin in fibroblastic cells [7, 19]. The precise biological functions of Hic-5 are not known, and in the present study we examined the effects of hydrogen peroxide on subcellular localization of Hic-5.

\section{Materials and Methods}

\section{Cell culture}

Mouse myoblastic $\mathrm{C} 2 \mathrm{C} 12$ cells were cultured in Dulbecco's modified MEM supplemented with 5\% fetal bovine serum and $10 \%$ horse serum. Mouse fibroblastic C3H10T1/2 and osteoblastic MC3T3 cells were cultured in Dulbecco's modified MEM supplemented with $10 \%$ fetal bovine serum. Plasmid DNA was transfected into cells using 


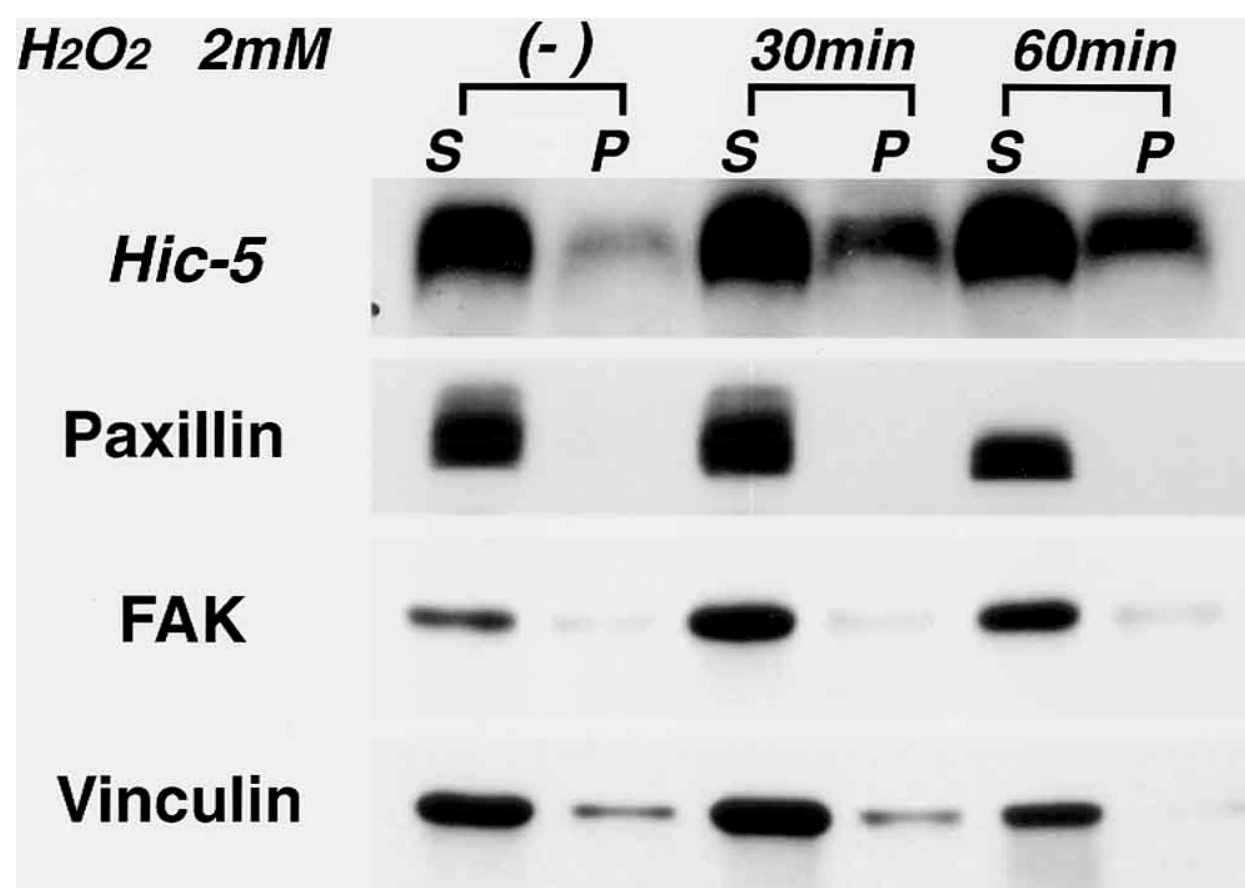

Fig. 1. Subcellular distribution of Hic-5 in C2C12 cells. Cells were either untreated or treated with 2 mM hydrogen peroxide for 30 and 60 min, and lysed in the presence of Triton X-100. Soluble (S) and insoluble (P) fractions were separated and Western blotted using the antibodies indicated.

Hic-5
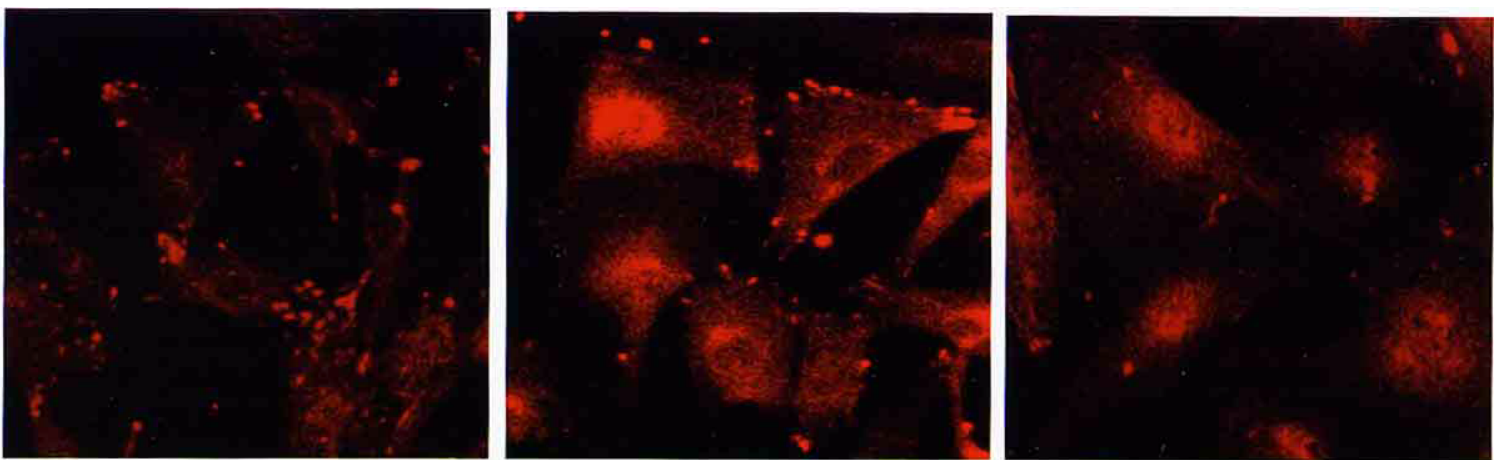

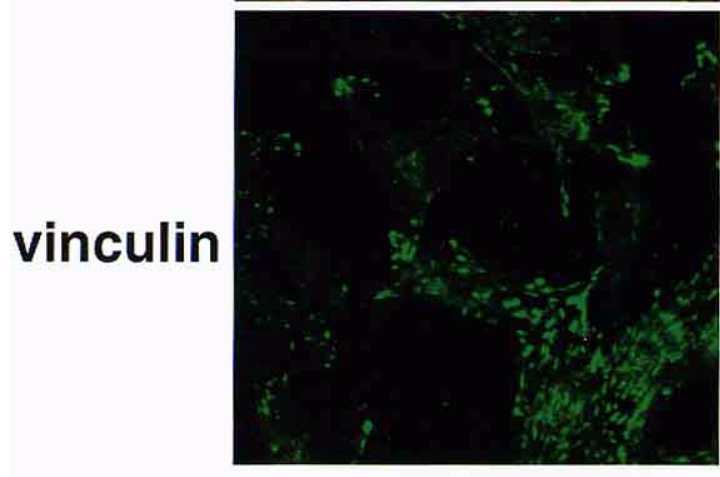

no treatment

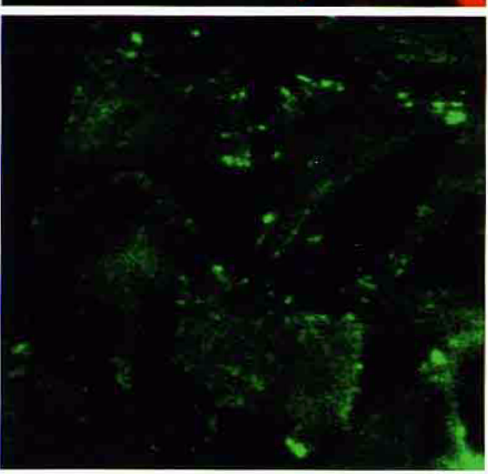

$10 \mathrm{~min}$

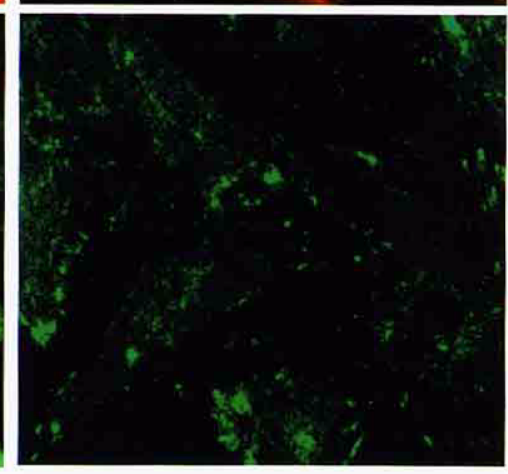

$30 \mathrm{~min}$

Fig. 2. Nuclear localization of Hic-5 in hydrogen peroxide-treated MC3T3 cells. Cells were treated with 0.5 mM hydrogen peroxide, fixed and stained with anti-Hic-5 or anti-vinculin antibody followed by the second fluorescently labeled antibody. 

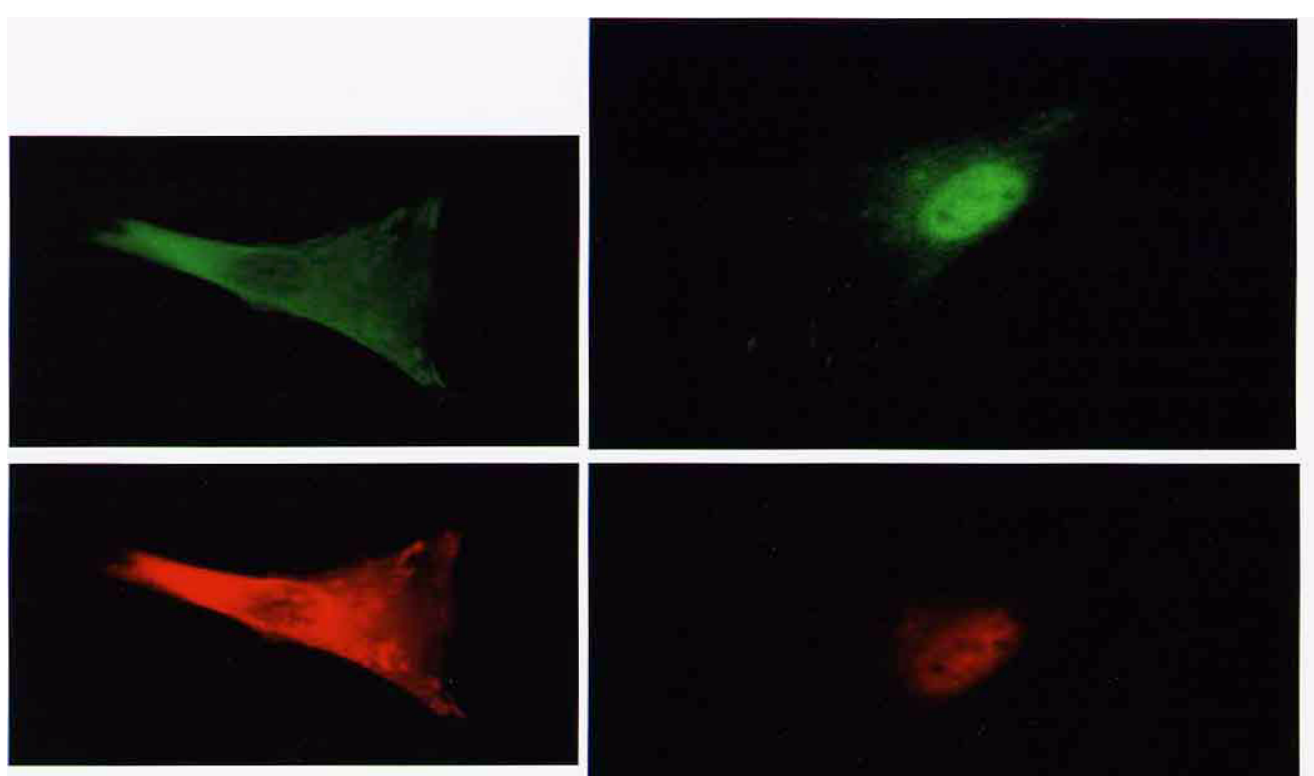

\section{anti-HA}

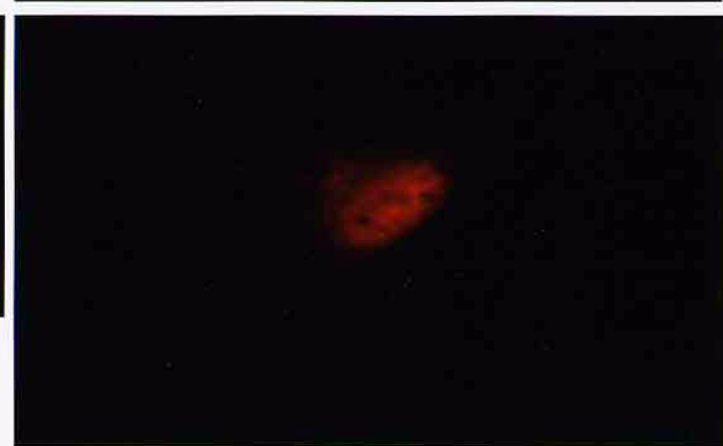

\section{anti-Hic-5}

\section{$0.5 \mathrm{mM} \mathrm{H} 2 \mathrm{O}_{2}(60 \mathrm{~min})$}

Fig. 3. Nuclear translocation of exogenously expressed Hic-5 in hydrogen peroxide-treated MC3T3 cells. Cells were transfected with pCGLD1mHic-5, and $24 \mathrm{hr}$ later treated with $0.5 \mathrm{mM}$ hydrogen peroxide for $60 \mathrm{~min}$, fixed and stained with anti-HA antibody.
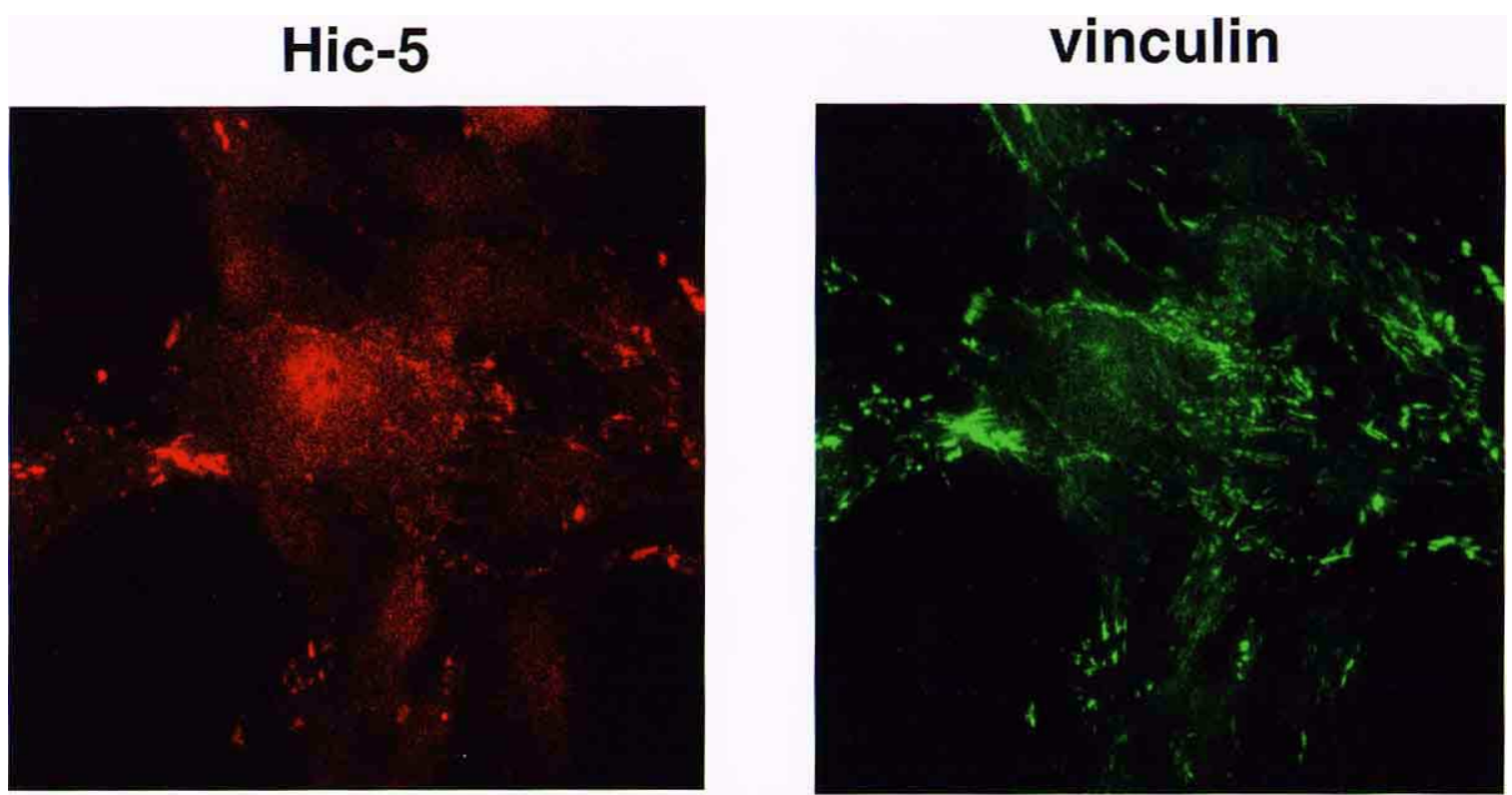

\section{leptomycinB 10ng/ml (2hr)}

Fig. 4. Effect of leptomycin B on subcellular localizaion of Hic-5. MC3T3 cells were treated with $10 \mathrm{ng} / \mathrm{ml}$ of leptomycin B for $2 \mathrm{hr}$, fixed and stained with anti-Hic-5 or anti-vinculin antibody. 

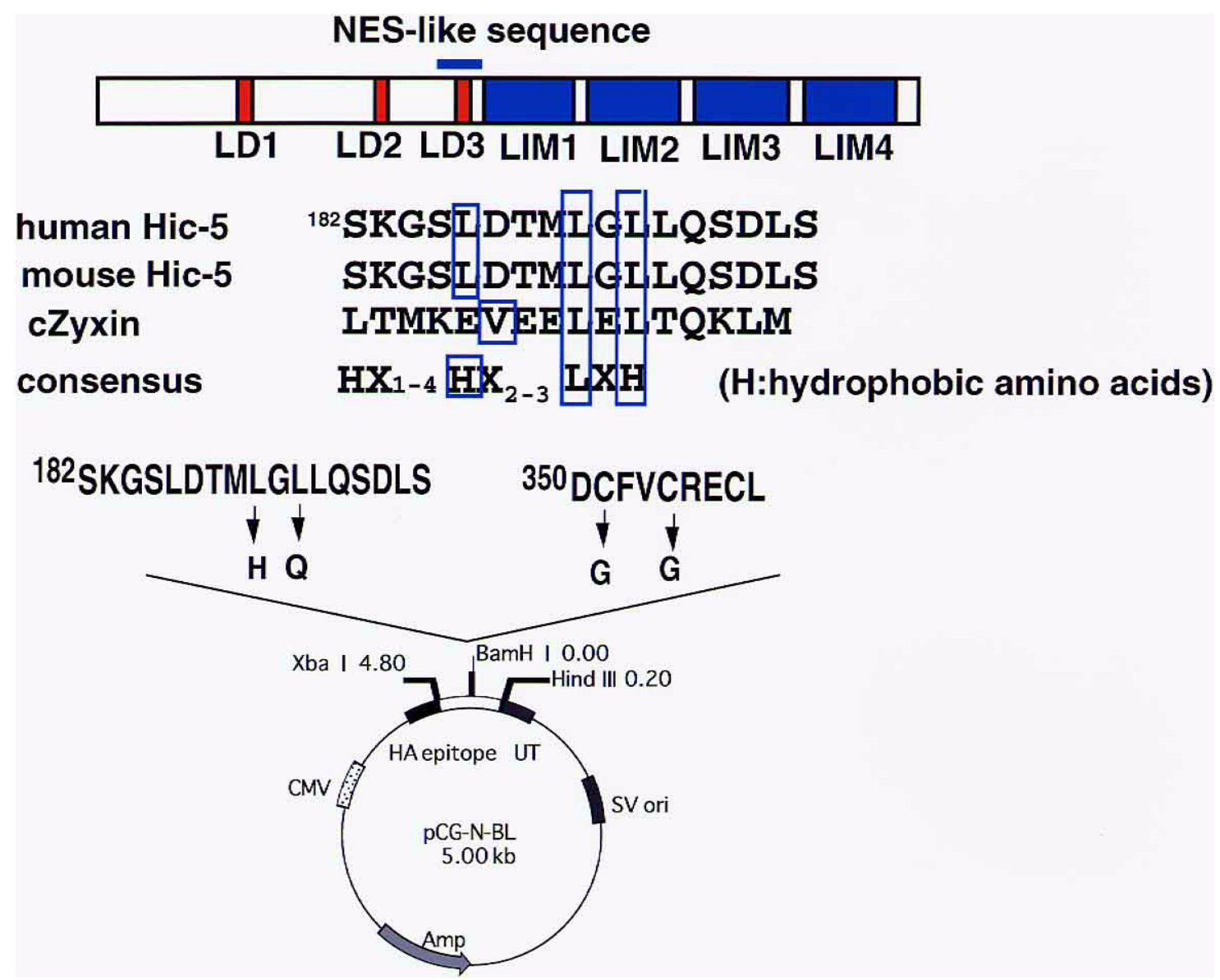

Fig. 5. Mutations introduced into Hic-5. Mutations at the nuclear export signal (NES)-like sequence and LIM3 domain were introduced by sitedirected mutagenesis, and expression vectors were constructed in pCG-N-BL. Consensus sequences of NES in Hic-5 are boxed. Two leucine residues in NES and two cysteine residues in LIM3 were mutated.

LipofectAMINE PLUS (Life Technologies, Inc) according to the manufacturer's protocol or by microinjection using a micromanipulator (Eppendorf).

\section{Plasmid construction}

pCG-LD1mhic-5 was the hemagglutinine (HA)-tagged mouse Hic-5 expression plasmid as described previously [13]. Nuclear export signal (NES)-like site (L191H, L193/ Q) and LIM 3 (C352G, C355/G) mutants of hic-5 were constructed by site-directed mutagenesis using a PCR-based kit (Mutan-Super, Takara, Co. Kyoto), and confirmed by sequencing.

\section{Subcellular fractionation}

Cells were lysed in a buffer containing $10 \mathrm{mM}$ Tris$\mathrm{HCl}, \mathrm{pH} 7.4,3 \mathrm{mM} \mathrm{MgCl} 2,10 \mathrm{mM} \mathrm{KCl}$, and $1 \%$ Triton $\mathrm{X}$ 100 on ice, and lysates were centrifuged at 10,000 rpm for 5 min in a Microfuge to separate the supernatant and pellet fractions. Samples were separated by SDS-PAGE and Western blotted using anti-Hic-5 (\#1024 [7]), anti-vinculin (Sigma Chemical Co.), anti-paxillin (Transduction Laboratories) or anti-FAK (Focal adhesion kinase, Transduction Laboratories) antibody.

\section{Immunostaining}

Transfected cells were fixed in $3.7 \%$ formaldehyde in phosphate buffered saline followed by permeabilization in $0.2 \%$ Triton $\mathrm{X}-100$ in saline. Cells were treated with antiHic-5, anti-vinculin or anti-HA (12CA5) antibody, and then treated with FITC-labeled monoclonal anti-mouse IgG or rhodamin-conjugated anti-rabbit IgG (Dako). Fluorescence microscopy was carried out using an Axioskope microscope (Carl Zeiss).

\section{Results and Discussion}

\section{Change in subcellular localization of Hic-5 following oxidative stress}

Cells were treated with non-toxic levels of hydrogen peroxide, and subcellular fractionation was carried out using C2C12 cells. The western blotting data shown in Fig. 1 indicated that most Hic-5 protein was recovered in the soluble fraction of Triton X-100-treated cell lysate, and only a small amount was detected in the pellet fraction of untreated cells. However, in cells treated with $2 \mathrm{mM}$ hydrogen peroxide for 30 to $60 \mathrm{~min}$ (Fig. 1), a significant increase in the level of Hic- 5 was observed in the pellet fraction. On the other hand, 

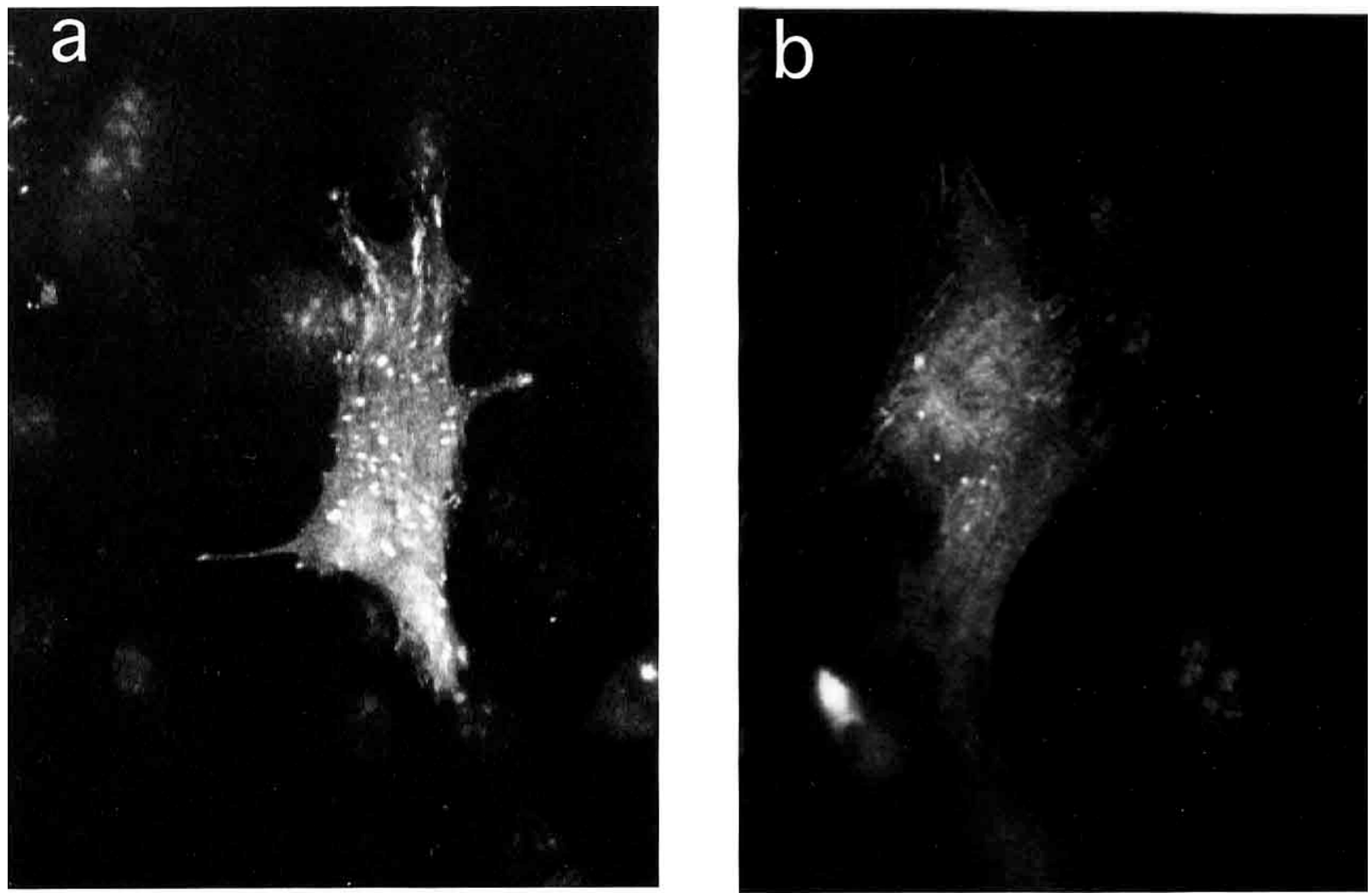

Fig. 6. Subcellular localization of Hic-5 mutants. Expression vectors of Hic-5 with mutation at the NES-like site (a) or NES-like site/LIM3 (b) were introduced into C3H10T1/2 cells, and cells were fixed and stained $24 \mathrm{hr}$ later.

the Hic-5 homologue, paxillin was detected only in the soluble fractions in cells treated with hydrogen peroxide.

Precise subcellular localization of Hic-5 under the above conditions was examined by immunocytochemistry with anti-Hic-5 antibody. MC3T3 cells were either untreated or treated with $0.5 \mathrm{mM}$ hydrogen peroxide, and immunostained using anti-Hic-5 antibody. The results shown in Fig. 2 indicated that Hic- 5 began to dislodge from the focal adhesions 10 min after treatment with hydrogen peroxide, and almost all Hic-5 was accumulated in the nuclei within 30 min after treatment. Vinculin, which was colocalized in the focal adhesion complex with Hic-5 in untreated cells, did not accumulate in the nuclei. These results indicated that nuclear Hic-5 accumulation was specific.

The accumulation of Hic-5 in the nuclei was further confirmed by transfection of HA-tagged Hic-5 expression vector and staining with anti-HA antibody (Fig. 3). Staining with anti-HA and anti-Hic-5 antibodies showed similar patterns, and hence the possibility was excluded that the results obtained using anti-Hic-5 antibody was due to crossreaction with other proteins.

\section{Nulcear export signal of Hic-5}

It was reported that oxidative stress affected cytoskeletal structure and cell-matrix interactions in cells from ocular tissue [21]. The change in the cytoskeletal structure may cause redistribution of focal adhesion protein. Hydrogen peroxide has been shown to regulate cytoplasmic localization of yeast transcription factor Pap1, and this process was dependent on the nuclear export factor Crm1 (exportin) [20].

Leptomycin B inhibits nuclear export of proteins through binding to Crm1 [2]. Treatment of MC3T3 cells with this agent resulted in nuclear accumulation of Hic-5, whereas vinculin remained at the focal adhesions (Fig. 4). This result indicates that Hic-5 contains a NES [12]. Examination of the amino acid sequence of Hic-5 revealed an NES-like sequence in the third LD domain (Fig. 5). Mutation was introduced in this NES-like sequence, and its subcellular localization was examined by immunofluorescent microscopy. The mutant protein, however, was localized mostly in the focal adhesions. We previously reported that the LIM3 domain participates in retention of Hic-5 in the focal adhesions [14]. Therefore, a mutation was also introduced at LIM3, and the double mutant exhibited nuclear localization, although the protein was not exclusively located in the nucleus (Fig. 6). It appears that release from the focal adhesion complex and association of Hic-5 with nuclear import factor are required for the nuclear translocation of Hic-5.

Many signaling molecules are known to translocate to the nucleus upon growth factor stimulation $[1,4-6,8,10]$. A focal adhesion protein, zyxin, shuttles beween the nucleus and cytoplasm [15], but the conditions that stimulate the shuttle have not been identified. Hic-5 is localized in focal 
adhesions in normal cells through interactions with other components of the focal adhesion complex [14], and oxidative stress may either directly or indirectly modify Hic-5 itself or interacting factors and permit its nuclear translocation. Further studies are necessary to determine the molecular mechanisms of the nuclear translocation of Hic-5.

\section{Acknowledgements}

Supported in part by a Grant-in-Aid for Scientific Research from the Ministry of Education, Sport, Culture, and, Science.

\section{References}

1. Behrens, J., von Kries, J. P., Kuhl, M., Bruhn, L., Wedlich, D., Grosschedl, R. and Birchmeier, W.: Functional interaction of beta-catenin with the transcription factor LEF-1. Nature 382; 638-642, 1996.

2. Fernerod, M., Ohno, M., Yoshida, M. and Mittag, I. W.: Crm1 is an export receptor for leucine-rich nuclear export signals. Cell 90; 1051-1060, 1997.

3. Finkel, T.: Oxygen radicals and signaling. Curr. Opinion Cell Biol. 10; 248-253, 1998.

4. Gottardi, C. J., Arpin, M., Fanning, A. S. and Louvard, D.: The junction associated protein zonula occludens-1, localizes to the nucleus before the maturation and during the remodeling of cellcell contact. Proc. Natl. Acad. Sci. U S A 93; 10779-10784, 1996.

5. Henderson, B. R.: Tumor suppressor APC protein shuttles between cytoplasm and nuclei. Nature Cell Biol. 2; 653-660, 2000.

6. Ihle, J. N.: STATs: Signal transducers and activators of transcription. Cell 84; 331-334, 1997.

7. Ishino, K., Kaneyama, J., Shibanuma, M. and Nose, K.: Specific decrease in the level of Hic-5, a focal adhesion protein, during immortalization of mouse embryonic fibroblasts, and its association with focal adhesion kinase. J. Cell. Biochem. 76; 411-419, 2000.

8. Khokhlatchev, A. V.: Phosphorylation of the MAP kinase ERK2 promottes its homodimerization and nuclear translocation. Cell 93; 605-615, 1998
9. Lander, H. M.: An essential role for free radicals and derived species in signal transduction. FASEB J. 11; 118-124, 1997.

10. Levievre, A. A. and Bissel, M. J.: Communication between the cell membrane and the nucleus. J. Cell. Biochem. 30/31; 250263, 1998.

11. Murata, M., Arata, S. and Nose, K.: Involvement of reactive oxygen species in the induction of chemokine JE/MCP-1 gene by phorbol-12-myristate-13-acetate in Balb 3T3 cells. Cell Struct. Funct. 22; 231-238, 1997.

12. Nigg, E. A.: Nucleocytoplasmic transport: signals, mechanisms and regulation. Nature 386; 779-787, 1997

13. Nishiya, N., Tachibana, K., Shibanuma, M., Mashimo, J. and Nose, K.: Hic-5 reduced cell spreading on fibronectin: Competitive effects between paxillin and Hic-5 through interaction with FAK. Mol. Cell. Biol. (in press).

14. Nishiya, N., Iwabuchi, Y., Shibanuma, M., Cote, J-F., Tremblay, M. L. and Nose, K.: Hic-5, a paxillin homologue, binds to the protein-tyrosine phosphatase PEST (PTP-PEST) through its LIM 3 domain. J. Biol. Chem. 274; 9847-9853, 1999.

15. Nix, D. A. and Beckerle, M. C.: Focal adhesion protein, zyxin, shuttles beween nuclei and cytoplasm. J. Cell Biol. 138; 11391147, 1997.

16. Ohba, M., Shibanuma, M., Kuroki, T. and Nose, K.: Production of hydrogen peroxide by transforming growth factor $\beta 1$ and its involvement in induction of egr-1 in mouse osteoblastic cells. $J$. Cell Biol. 269; 1079-1088, 1994.

17. Shibanuma, M., Mashimo, J., Kuroki, T. and Nose, K.: Characterization of the TGF $\beta 1$-inducible hic-5 gene that encodes a putative novel zinc finger protein and its possible involvement in cellular senescence. J. Biol. Chem. 269; 26767-26774, 1994.

18. Sundaresan, M., Yu, Z.-X., Ferrans, V. J., Irani, K. and Finkel, T.: Requirement for generation of $\mathrm{H}_{2} \mathrm{O}_{2}$ for platelet-derived growth factor signal transduction. Science 270; 296-299, 1995.

19. Thomas, S. M., Hagel, M. and Turner, C. E.: Characterization of a focal adhesion protein, Hic-5, that shares extensive homology with paxillin. J. Cell Sci. 112; 181-190, 1999.

20. Toone, W. M., Kuge, S., Samuels, M., Morgan, B. A., Toda, T. and Jones, N.: Regulation of the fission yeast transcription factor Pap1 by oxidative stress: requirement for the nuclear export factor Crm1 (exportin) and the stress-activated MAP kinase Sty $1 /$ Spc1. Genes \& Develop. 12; 1453-1463, 1998.

21. Zhou, L., Li, Y. and Yue, B. Y. J. T.: Oxidative stress affects cytoskeletal structure and cell-matrix interactions in cells from an ocular tissue. J. Cell. Physiol. 180; 182-189, 1999. 